ACTA MYCOLOGICA

Vol. 48 (1): 61-71

2013

DOI: $10.5586 / \mathrm{am} .2013 .008$

\title{
Species diversity of yeast-like fungi in some eutrophic lakes in Olsztyn
}

\author{
ANNA BIEDUNKIEWICZ*, MARIA DYNOWSKA, ELŻBIETA EJDYS \\ and EWA SUCHARZEWSKA
}

\author{
Department of Mycology, Faculty of Biology and Biotechnology \\ University of Warmia and Mazury in Olsztyn \\ Oczapowskiego 1A, PL-10-917 Olsztyn, *corresponding author: alibi@uwm.edu.pl
}

Biedunkiewicz A., Dynowska M., Ejdys E., Sucharzewska E.: Species diversity of yeast-like fungi in some eutrophic lakes in Olsztyn. Acta Mycol. 48 (1): 61-71, 2013.

The study is part of hydromycological investigations of man-affected water bodies in Olsztyn. Our results show that yeast-like fungi are permanent components in three selected lakes (lakes Tursko, Długie and Skanda). Their abundance and species composition depend on the trophic state of each lake.

Key words: yeast-like fungi, eutrophic lakes, water ecosystem, monitoring studies

\section{INTRODUCTION}

Long-term mycological monitoring studies conducted in a variety of water ecosystems have shown that microfungi are a permanent component of microbiota in inland waters whereas each increase in the abundance is mostly related to growing anthropopressure and progressive eutrophication processes (Dynowska 1995; Dynowska et al. 2001; Biedunkiewicz 2007; Biedunkiewicz et al. 2007; Biedunkiewicz, Baranowska 2011). While a vast majority of lakes in north-east Poland, including lakes in Olsztyn, are eutrophic, the trophic state of some lakes is relatively low or periodically variable.

Although Olsztyn has as many as 11 lakes mycological characteristics of only a few have examined. The aim of this study was to determine the taxonomic diversity of fungi in three lakes in Olsztyn (Tyrsko ${ }^{\star}$, Długie and Skanda ${ }^{\star}$ ) which differ by morphometry, natural resistance to degradation, eutrophication level and natural values. 


\section{STUDY AREA}

The lakes lie along the line dividing Olsztyn into two almost equal parts from the north-west (Tyrsko) towards the south-east (Skanda) with lake Długie being positioned centrally (Fig. 1). Morphometric and physicochemical features of the three lakes are given in Table 1.

Lake Tyrsko (Żbik) is in north-west Olsztyn. It is rhomboid in shape and its shoreline is poorly developed. The water surface is small but the maximum depth is high. The lake is a closed reservoir and does not have any tributaries or outflows. It was a natural reserve between 1957 and 1987 to protect Isöetes lacustris L. rarely occurring in the Masurian Lakeland (Lossow et al. 2005). Lake Tyrsko is thought to be in a transition phase from the b-mesotrophic to eutrophic type due to a small load of phosphorus and nitrogen (Tab. 1).

Lake Długie is located in the western part of Olsztyn in the Eyna-Pregoła river basin. It is elongated from the north southwards. Three pools can be distinguished based on the shape of the lake basin. They differ by surface, maximum depth and

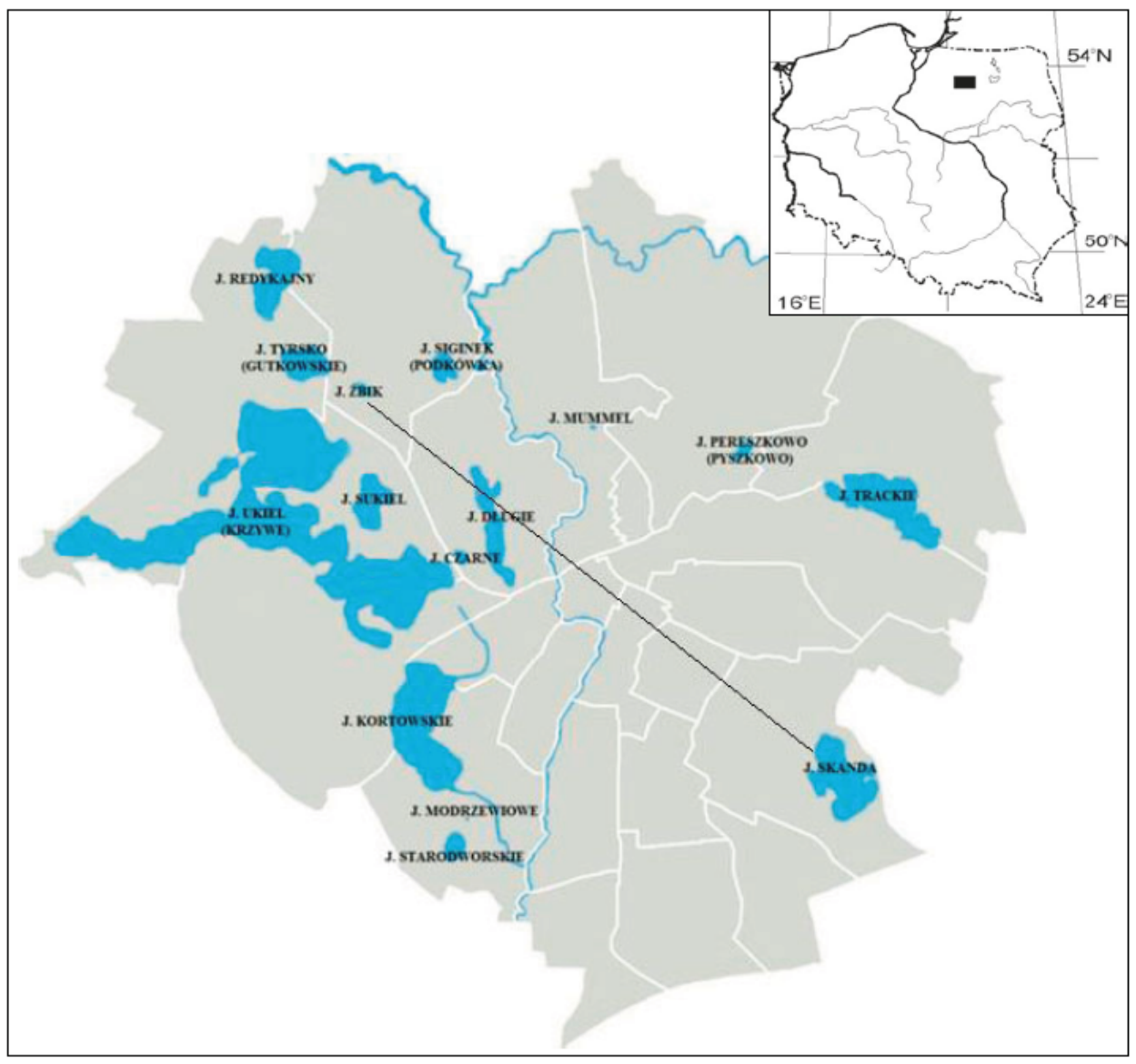

Fig. 1. The north-west system of the lakes towards the south-east. 
Table 1

Important morphometric features, degradation degree, phosphorus load and purity class of the three lakes in Olsztyn (based on Lossow et al. 2005) with number of species and genera in individual seasons and the lakes' trophic state

\begin{tabular}{|c|c|c|c|c|c|c|c|c|c|c|}
\hline 营 & 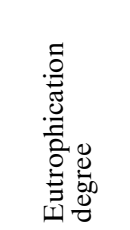 & 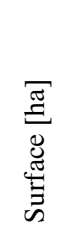 & 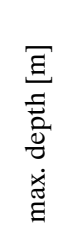 & 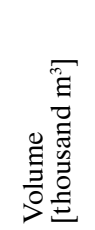 & 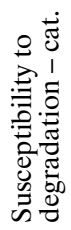 & 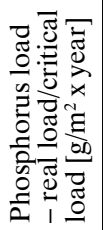 & 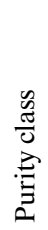 & 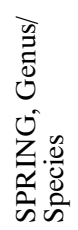 & 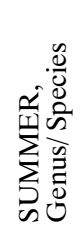 & 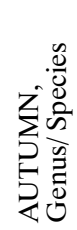 \\
\hline Tyrsko & low & 18.6 & 30.4 & 1786.1 & II & $\begin{array}{l}0.120 / \\
0.2\end{array}$ & II & $32 / 19$ & $10 / 8$ & $9 / 8$ \\
\hline Długie & moderate & 26.8 & 17.3 & 1414.8 & II & $\begin{array}{l}0.171 / \\
0.136\end{array}$ & II & $28 / 21$ & $28 / 18$ & $13 / 11$ \\
\hline Skanda & high & 51.1 & 12 & 2963.8 & II & $\begin{array}{l}0.106 / \\
0.142\end{array}$ & III & $13 / 10$ & $11 / 9$ & $13 / 10$ \\
\hline
\end{tabular}

development of the shoreline. The southern pool lies in a strongly urbanized part, the central pool borders on buildings to the west and is forested on the eastern side, and the northern part is fully surrounded by the forest. Lake Długie is a hydrologically closed reservoir. It is supplied by run-offs from a small basin and run-off waste water from a residential estate. As the sanitary condition of the lake was poor and a complete oxygen deficiency was reported, an attempt was made to recultivate the lake by flushing in 1983. An artificial connection between lakes Ukiel and Czarne by an open ditch was made and lakes Czarne and Długie were connected (by an underground collector). This did not improve water quality and recultivation by aeration with destratification began in 1987. Aeration was carried out in two stages: the first covered the period from 1987 until 1990 and the second from 1991 until 2000. Artificial, long-term aeration of the lake considerably improved environmental conditions but water quality was still outside water quality categories after aerators had been switched off. Phosphorus precipitation from water and phosphorus inactivation in bottom sediments were used in 2001-2003. The treatment was conducted three times and water purity class II was recorded (Lossow et al. 2005). At present a moderately eutrophic state is recorded in the lake (Tab. 1).

Lake Skanda lies in the south-eastern part of Olsztyn by the Olsztyn-Szczytno trunk road in the Łyna-Pregoła river basin. The lake is relatively shallow (Tab. 1) and its shoreline is quite well developed with a few bays and an island in the western part. The shores are surrounded by the forest lying on a high hill on the western and southern side. A former waste disposal site for Olsztyn is situated on the other side of the lake. The lake's catchment is agricultural and forested, partly urbanized, with several residential buildings that are not connected to the municipal waste system. A guarded bathing beach and a sandy unsupervised beach lie on the western shore of the lake. An inflow of contaminants has degraded waters to purity class III and organic compounds, mostly biogenic, must occur permanently in the lake as the level of eutrophication is high. Astatic reservoirs located in the vicinity play an important role of a buffer and a filter that retain the majority of contaminants (Dynowska et al. 2005). 


\section{MATERIAL AND METHODS}

Investigations were conducted in 2011 every month from early spring $(04 / 05)$ until late autumn (09/10). Yeast-like fungi sampled from the littoral zone of the lakes were analyzed. The number of study sites correlated with the size of each water body: Tyrsko -4 sites, Długie -6 sites and Skanda -10 sites. The sites were distributed around the lakes.

Samples $(250 \mathrm{ml})$ were collected into sterilized bottles using an extension arm from a depth of up to $30 \mathrm{~cm}, 4.5 \mathrm{~m}$ away from the shore. The membrane filter method was used to isolate and determine the number of fungal colonies. $100 \mathrm{ml}$ of water was passed through a Millipore membrane filter, $0.45 \mu \mathrm{m}$ pore size, using a PL 2/2 vacuum pump (0.095 MPa). The pressure did not exceed 0.2 bar. The filter was placed in a beaker with $20 \mathrm{ml}$ of $0.9 \%$ sterile $\mathrm{NaCl}$ and centrifuged for $0.5 \mathrm{~h}$ (Biedunkiewicz 2007). The solution (1 ml) was collected from each beaker with a sterile pipette and placed on a Petri dish with solid Sabouraud medium containing $0.5 \mathrm{~g}$ of chloramphenicol. The solution was spread on the dish with a sterile "hockey stick". The filter from each beaker was placed on Sabouraud medium to isolate yeast-like fungi retained in the filter mesh. Inoculations were incubated for $48-72 \mathrm{~h}$ at $37^{\circ} \mathrm{C}$. Colonies of yeast-like fungi were counted and examined microscopically (colour, consistency, the structure of the colony's margin, odour). Colonies were next inoculated on Sabouraud slants and incubated again for $48-72$ hours at $37^{\circ} \mathrm{C}$. Species identification was based on macroscopic and microscopic features and biochemical properties (zymograms and auxanograms). Microscopic features of fungi were analyzed in microcultures conducted on Nickerson agar. Microcultures were incubated between 78 and $144 \mathrm{~h}$ at $37^{\circ} \mathrm{C}$ (Biedunkiewicz-Ziomek, Dynowska 2004).

Fungi were determined using keys by Lodder, Kreger-van Rij (1967), Kurtzman, Fell (2000) and Kurtzman et al. (2011). Photographic documentation was made. Strains of fungi were catalogued and deposited in the collection of the Department of Mycology, Faculty of Biology and Biotechnology, University of Warmia and Mazury in Olsztyn.

\section{RESULTS}

A total of 120 samples were collected from 20 sites in the period between late April/ early May and late September/late October 2011. Altogether 124 isolates of yeastlike fungi were obtained. Eighty four species of fungi belonging to 39 genera were identified. Seventy two isolates belonging to 49 genera were recorded in lake Tyrsko, 39 isolates of 40 genera were recorded in lake Długie, and 34 isolates of 28 genera were recorded in lake Skanda. Fungi of the genus Candida were the most frequently recorded species (16 species). Fungi of the genera Kazachstania and Kluyveromyces (five species each) and Wickerhamomyces (three species) were recorded less frequently (Tab. 2).

Among all species of yeast-like fungi, five were classified as potential pathogens, 52 are saptrophs and 27 are fungi that are saptrotrophs or pathogens depending on 
the biocoenotic system and bioecological conditions. A few species take part in selfpurification processes or are pollution indicators.

The greatest number of fungi were recorded in spring in a low trophic state lake (32 species), slightly fewer in summer in low and moderate trophic state lakes (28 species in each) and the smallest number in spring in a highly eutrophized lake (only nine species). The species diversity in autumn was similar across the three lakes. A detailed analysis of the species richness in the waters of the three lakes shows that the greatest diversity is observed in lake Tyrsko and the smallest in the eutrophized lake Skanda (Tab. 1).

Fungi of the genus Candida (seven species from each lake) were isolated from all the lakes. Fungi of the genus Kluyveromyces (5 species) were often recorded in lake Tyrsko.

Fungi of 13 genera were recorded from spring to autumn regardless of the trophic state (Fig. 2). Those incuded Candida albicans, Candida solanii, Debaryomyces hansenii, Kluyveromyces lactis var. lactis, Lachancea thermotolerans, Metschnikowia pulcherrima, Meyerozyma guilliermondii, Pichia membranifaciens, Saccharomyces cerevisiae, Wanderwaltozyma polyspora, Wanderwaltozyma yarrowii and Wickerhamomyces bisporus. They are both pathogens and harmless saprotrophs (Tab. 2).

Table 2

Species of fungi isolated from the lakes

\begin{tabular}{|c|c|c|c|c|c|c|c|c|c|c|}
\hline \multirow[t]{2}{*}{ Species } & \multicolumn{3}{|c|}{ Tyrsko } & \multicolumn{3}{|c|}{ Długie } & \multicolumn{3}{|c|}{ Skanda } & \multirow[t]{2}{*}{ Ecology } \\
\hline & $\mathrm{Sp}$ & $\mathrm{Su}$ & A & $\mathrm{Sp}$ & $\mathrm{Su}$ & A & $\mathrm{Sp}$ & $\mathrm{Su}$ & A & \\
\hline $\begin{array}{l}\text { Brettanomyces bruxellensis Kufferath \& van Lear } \\
\text { (1921) (anamorf.) }\end{array}$ & & & & + & & & & & + & sap \\
\hline $\begin{array}{l}\text { B. nanus (M. Th. Smith, Batenburg-van Vegte \& } \\
\text { Sheffers) Boekhout et al.. } 1994\end{array}$ & & + & & & & & & & & sap \\
\hline $\begin{array}{l}\text { Candida aaseri Dietrichson ex van Uden \& H.R. } \\
\text { Bucley (1970) }\end{array}$ & + & & & & & & & & & path \\
\hline C. albicans (Robin) Berkhout (1923) & + & + & & & + & & + & & + & path \\
\hline $\begin{array}{l}\text { C. albicans (Robin) Berkhout (1923) var. } \\
\text { stellatoidea (Jones \& Martin) Didens \& Lodder } \\
\text { (1942) }\end{array}$ & & & & & & & & + & & path \\
\hline C. catenulata Diddens \& Lodder (1942) & & & & & + & & & & & path \\
\hline $\begin{array}{l}\text { C. glabrata (Anderson) S.A. Myer \& Yarrow } \\
\text { (Yarrow \& Meyer 1978) }\end{array}$ & & & & & & & + & + & & sap/path \\
\hline C. glaebosa Komagata \& Nakase (1965) & & & & & & & & & + & sap \\
\hline $\begin{array}{l}\text { C. intermedia (Ciferri \& Ashford) Langeron \& } \\
\text { Guerra (1938) }\end{array}$ & & & & & + & & & & & sap \\
\hline $\begin{array}{l}\text { C. kruissii (Kocková-Kratochvílová \& } \\
\text { Ondrušová) S.A. Meyer \& Yarrow (1978) }\end{array}$ & + & & & & & & & & & sap \\
\hline $\begin{array}{l}\text { C. lipolytica (F.C. Harrisson) Diddens \& Lodder } \\
\text { (1942) (anamorf.) }\end{array}$ & & & & & & & & + & & sap/path \\
\hline C. melibiosica H.R. Bucley \& van Uden (1968) & & + & & & & & & + & & sap/path \\
\hline $\begin{array}{l}\text { C. parapsilosis (Ashford) Longeron \& Talice } \\
\text { (1932) }\end{array}$ & + & & & & + & & & & & sap/path \\
\hline C. saitoana Nakase \& M. Suzuki (1985 b) & & & & & & + & & & & sap \\
\hline $\begin{array}{l}\text { C. sake (Saito \& Oda) van Uden \& H.R. Bucley } \\
\text { ex S.A. Meuer \& Ahearn (1983) }\end{array}$ & & & & & + & & & & & sap \\
\hline C. silvae Vidal - Leiria \& van Uden (1963) & + & & & & & & & & & sap/path \\
\hline C. solani Lodder \& Kreger-van Rij (1952) & + & + & + & & + & + & & & & sap \\
\hline C. tropicalis (Castellani) Berkhout (1923) & & & & & + & & & + & & $\begin{array}{l}\text { sap/path/ } \\
\text { ind/pur }\end{array}$ \\
\hline
\end{tabular}


Table 2 - cont.

\begin{tabular}{|c|c|c|c|c|c|c|c|c|c|c|}
\hline $\begin{array}{l}\text { Citeromyces matritensis (Santa María) Santa } \\
\text { María (1957) }\end{array}$ & & + & & & & & & & & sap \\
\hline $\begin{array}{l}\text { Cyniclomyces guttulatus (Robin) van der Walt \& } \\
\text { D.B. Scott (1971) }\end{array}$ & & & & & & & & & + & path \\
\hline Debaryomyces fabryi M. Ota (1924b) & & & + & & & & & & & path \\
\hline $\begin{array}{l}\text { D. hansenii (Zopf) Lodder \& Kreger-van Rij } \\
\text { (1952) = Candida famata (anamorf.) (Harrison) } \\
\text { S.A. Meyer \& Yarrow }\end{array}$ & + & + & + & + & + & & & & + & $\begin{array}{l}\text { sap/path/ } \\
\text { pur }\end{array}$ \\
\hline D. maramus di Menna (1954) & + & & & & & & & & & sap/path \\
\hline $\begin{array}{l}\text { Dipodascus armillariae W. Gams (1983) } \\
\text { (teleomorf.) }\end{array}$ & & + & & & + & & & & & sap \\
\hline $\begin{array}{l}\text { Hanseniaspora osmophila (Niehaus) Phaff, M.W. } \\
\text { Miller \& Schifrine ex M.Th. Smith (1984) }\end{array}$ & & + & & & & & & & & sap \\
\hline $\begin{array}{l}\text { H. uvarum (Niehaus) Shehata, Mrak \& Phaff ex } \\
\text { M. Smith (1984) }\end{array}$ & & & & & & & & & & sap \\
\hline $\begin{array}{l}\text { Kazachstania africana (van der Walt) Kurtzman } \\
(2003)\end{array}$ & + & & & & & & & & & sap \\
\hline $\begin{array}{l}\text { K. exigua (Reess ex E. C. Hansen) Kurtzman } \\
(2003)\end{array}$ & & & & & + & & & & & sap \\
\hline $\begin{array}{l}\text { K. exigua (Reess ex E.C. Hansen) Kurtzman } \\
(2003)=\text { Candida holmii (anamorf.) }\end{array}$ & + & & & & & & & & & sap \\
\hline $\begin{array}{l}\text { K. lodderae (van der Walt \& Tscheuschner) } \\
\text { Kurtzman (2003) }\end{array}$ & & + & & & & & & & & sap \\
\hline K. transvaalensis (van der Walt) Kurtzman (2003) & & + & & & & & & & & sap \\
\hline $\begin{array}{l}\text { Kloeckera africana (anamorf.) (Klöcker) Janke } \\
\text { (1928) }\end{array}$ & + & & & & & & & & & sap \\
\hline $\begin{array}{l}\text { Kluyveromyces aestuarii (Fell) van der Walt } \\
\text { (1971) }\end{array}$ & + & + & & & + & & & & & sap \\
\hline K. lactis (Dombrowski) van der Walt (1971) & + & + & & + & + & & & & + & sap \\
\hline $\begin{array}{l}\text { K. lactis var. lactis (Dombrowski) van der Walt } \\
(1986)\end{array}$ & & & + & + & & & & & & sap \\
\hline K. marxianus (E. C. Hansen) van der Walt (1971) & + & + & & + & + & & & & & sap/path \\
\hline $\begin{array}{l}\text { K. wickerhamii (Phaff, M.W. Miller \& Shifrine) } \\
\text { van der Walt (1971) }\end{array}$ & + & & + & & & & & & & sap \\
\hline Lachancea cidri (Legakis) Kurtzman (2003) & & & & & + & & & & & sap \\
\hline L. thermotolerans (Filippov) Yarrow (2003) & + & + & + & & + & & & & & sap \\
\hline L. waltii (K. Kodama) Kurtzman (2003) & + & + & & & & & & & & sap \\
\hline $\begin{array}{l}\text { Lindnera jadinii (A. \& R. Sartory, Weill \& J. } \\
\text { Meyer) Robnett \& Basehoar - Powers }(2008)= \\
\text { Candida utilis (anamorf.) }\end{array}$ & + & + & & + & & & & + & & sap/path \\
\hline $\begin{array}{l}\text { Lipomyces lipofer Lodder \& Kreger-van Rij ex } \\
\text { Slooff (1970a) }\end{array}$ & & & + & & & & + & & & sap/path \\
\hline $\begin{array}{l}\text { Lodderomyces elongisporus (Recca \& Mrak) van } \\
\text { Walt (1971) }\end{array}$ & + & & & & & & & & & sap/path \\
\hline $\begin{array}{l}\text { Magnusiomyces capitatus (de Hoog, M.Th. Smith } \\
\text { \& Guého) de Hoog \& M.Th. Smith (2004) }\end{array}$ & & & & & & & & + & & sap/path \\
\hline $\begin{array}{l}\text { Metschnikowia pulcherrima Pitt \& M.W. Miller } \\
\text { (1968) = Candida pulcherrima (anamorf.) } \\
\text { (Lindner) Windisch (1940) }\end{array}$ & + & + & + & & & & & + & & sap/path \\
\hline M. reukaufii Pitt \& M. W. Miller (1968) & & & & & + & & & & & sap \\
\hline $\begin{array}{l}\text { Meyerozyma guilliermondii (Wickerham) } \\
\text { Kurtzman \& M. Suzuki (2010) = Candida } \\
\text { guilliermondii (anamorf.) (Castellani) Berkhout } \\
\text { (1923) }\end{array}$ & + & + & & + & + & + & + & & & $\begin{array}{l}\text { sap/path/ } \\
\text { ind }\end{array}$ \\
\hline $\begin{array}{l}\text { Millerozyma farinosa Kurtzman \& M. Suzuki } \\
(2010)\end{array}$ & & & & & + & & + & & & sap/path \\
\hline $\begin{array}{l}\text { Moniliella mellis (Fabian \& Quinet) V. Rao \& de } \\
\text { Hoog (1975) }\end{array}$ & & & & & + & & & & & sap \\
\hline $\begin{array}{l}\text { M. spathulata (de Hoog) Rosa \& Lachance } \\
\text { (Rosa et al.. (2008) }\end{array}$ & & & & & & & + & & & sap \\
\hline $\begin{array}{l}\text { Mrakiella aquatica (Jones \& Sloff) Margesin \& } \\
\text { Fell (2008) }\end{array}$ & & & & + & & & & & & sap \\
\hline
\end{tabular}


Table 2 - cont.

\begin{tabular}{|c|c|c|c|c|c|c|c|c|c|c|}
\hline $\begin{array}{l}\text { Nakaseomyces bacillisporus ( Lachance, Phaff \& } \\
\text { Starmer) Kurtzman (2003) }\end{array}$ & + & & & & & & & & & sap \\
\hline $\begin{array}{l}\text { Nakazawaea holstii (anamorf.) (Wickerham) Y. } \\
\text { Yamada, Maeda \& Mikata (1994c) }\end{array}$ & & + & & & & & & & & sap/path \\
\hline $\begin{array}{l}\text { Naumovozyma castellii (Capriotti) Kurtzman } \\
\text { (2008) }\end{array}$ & & & & & & + & & & & sap \\
\hline $\begin{array}{l}\text { Pichia kudriavzevii Boidin, Pignal \& Besson } \\
\text { (1965) = Candida krusei (anamorf.) }\end{array}$ & & + & & & & & & + & + & sap/path \\
\hline $\begin{array}{l}\text { P. membranifaciens (E. C. Hansen) E. C. Hansen } \\
(1904)\end{array}$ & + & + & & + & + & & & & + & $\begin{array}{c}\text { sap/path/ } \\
\text { pur }\end{array}$ \\
\hline $\begin{array}{l}\text { Priceomyces carsonii (Phaff \& Knapp) M. Suzuki } \\
\text { \& Kurtzman }(2010)\end{array}$ & + & + & & & + & & & & & sap \\
\hline $\begin{array}{l}\text { Rhodosporidium diobovatum Newell \& I. L. } \\
\text { Hunter (1970) }\end{array}$ & & & & & + & + & & & & sap \\
\hline Rh. lusitaniae Á Fonseca \& Sampaio (1992) & + & & & & & & & & & sap \\
\hline Rh. toruloides Banno (1967) & & & & & & + & & & & sap \\
\hline $\begin{array}{l}\text { Saccharomyces cerevisiae Meyen ex E. C. Hansen } \\
\text { (1883) }\end{array}$ & & + & & & & + & & + & & sap/path \\
\hline S. ludwigii E.C. Hansen (1904) & & & & & & & & + & & sap/path \\
\hline Saccharomycopsis capsularis Schiönning (1903) & & & & & & + & + & + & + & sap/path \\
\hline $\begin{array}{l}\text { S. fermentans (C.-F. Lee, F.-L.Lee, Hsu \& Phaff) } \\
\text { Kurtzman \& Robnett (1995) }\end{array}$ & & & & & + & & & & & sap \\
\hline $\begin{array}{l}\text { S.s javanensis (Klöcker) Kurtzman \& Robnett } \\
\text { (1995) }\end{array}$ & & & & & & & & & + & sap \\
\hline $\begin{array}{l}\text { Schefferomyces segobiensis (Santa María \& } \\
\text { García Aser) Kurtzman \& M. Suzuki (2010) }\end{array}$ & & & + & & & & & & & sap \\
\hline $\begin{array}{l}\text { Schizosaccharomyces octosporus Beijerinck } \\
\text { (1894) }\end{array}$ & + & & & & & & & & & sap \\
\hline Schwanniomyces occidentalis Klöcker (1909a) & + & & & & & & & & & sap \\
\hline $\begin{array}{l}\text { Sch. polymorphus (Klöcker) M. Suzuki \& } \\
\text { Kurtzman (2010) }\end{array}$ & + & & & & & & + & & & sap \\
\hline $\begin{array}{l}\text { Sch. pseudopolymorphus (C. Ramírez \& Boidin) } \\
\text { M. Suzuki \& Kurtzman (2010) }\end{array}$ & & & & & & + & & & & sap \\
\hline $\begin{array}{l}\text { Sugiyamaella smithiae (Giménez-Jurado) } \\
\text { Kurtzman \& Robnett (2007b) }\end{array}$ & & & & & & + & & & & sap \\
\hline $\begin{array}{l}\text { Tetrapisispora phaffi (van der Walt) Ueda - } \\
\text { Nishimura \& Mikata (1999) }\end{array}$ & + & + & + & & & & & & & sap \\
\hline Torulaspora delbruecki (Lindner) Lindner (1904) & & & & & & & & & + & sap/path \\
\hline $\begin{array}{l}\text { Trichomonascus cyferrii (M. Th. Smith, van der } \\
\text { Walt \& E. Johannsen) Kurtzman \& Robnett } \\
\text { (2007) }\end{array}$ & & & & & & + & & & & sap/path \\
\hline $\begin{array}{l}\text { T. farinosus (de Hoog, Rantio-Lehtimäki \& M. } \\
\text { Th. Smith) Kurtzman \& Robnett (2007) (1985) }\end{array}$ & & & & & + & & & & & sap \\
\hline Wanderwaltozyma polyspora Kurtman (2003) & + & + & + & & + & & & & & sap \\
\hline $\begin{array}{l}\text { W. yarrowii (van der Walt, E. Johansen, } \\
\text { Opperman \& Halland) Kurtzman (2003) }\end{array}$ & + & + & + & & & & & & & sap \\
\hline $\begin{array}{l}\text { Wickerhamomyces anomalus (E. C. Hansen) } \\
\text { Kurtzman, Robnett \& Basehoar - Power (2008) }\end{array}$ & & & & & + & & & & & sap/path \\
\hline $\begin{array}{l}\text { W. bisporus (Beck) Kurtzman, Robnett \& } \\
\text { Basehoar - Powers (2008) }\end{array}$ & & + & & & + & & + & + & + & sap/path \\
\hline $\begin{array}{l}\text { W. bovis (van Uden \& de Carmo - Sousa) } \\
\text { Kurtzman, Robnett \& Basehoar - Powers (2008) }\end{array}$ & + & & & & & & & & & sap \\
\hline $\begin{array}{l}\text { Yamadazyma akitaensis (K. Kodama) Kurtzman } \\
\text { \& M. Suzuki (2010) }\end{array}$ & & & + & & & & & & & sap \\
\hline $\begin{array}{l}\text { Zygosaccharomyces baiili (Lindner) } \\
\text { Guilliermond (1912) }\end{array}$ & & & & & + & & & & & sap/path \\
\hline Z. mellis Fabian \& Quinet (1928) & & & & & & & & & + & sap \\
\hline $\begin{array}{l}\text { Z. rouxii (Boutroux) Yarrow (von Arx et al.) } \\
\text { (1977b) }\end{array}$ & & + & & & & & & & & sap \\
\hline
\end{tabular}

Legend: sap - saprotrophic, path - pathogenic, ind - indicator, pur - purifying 


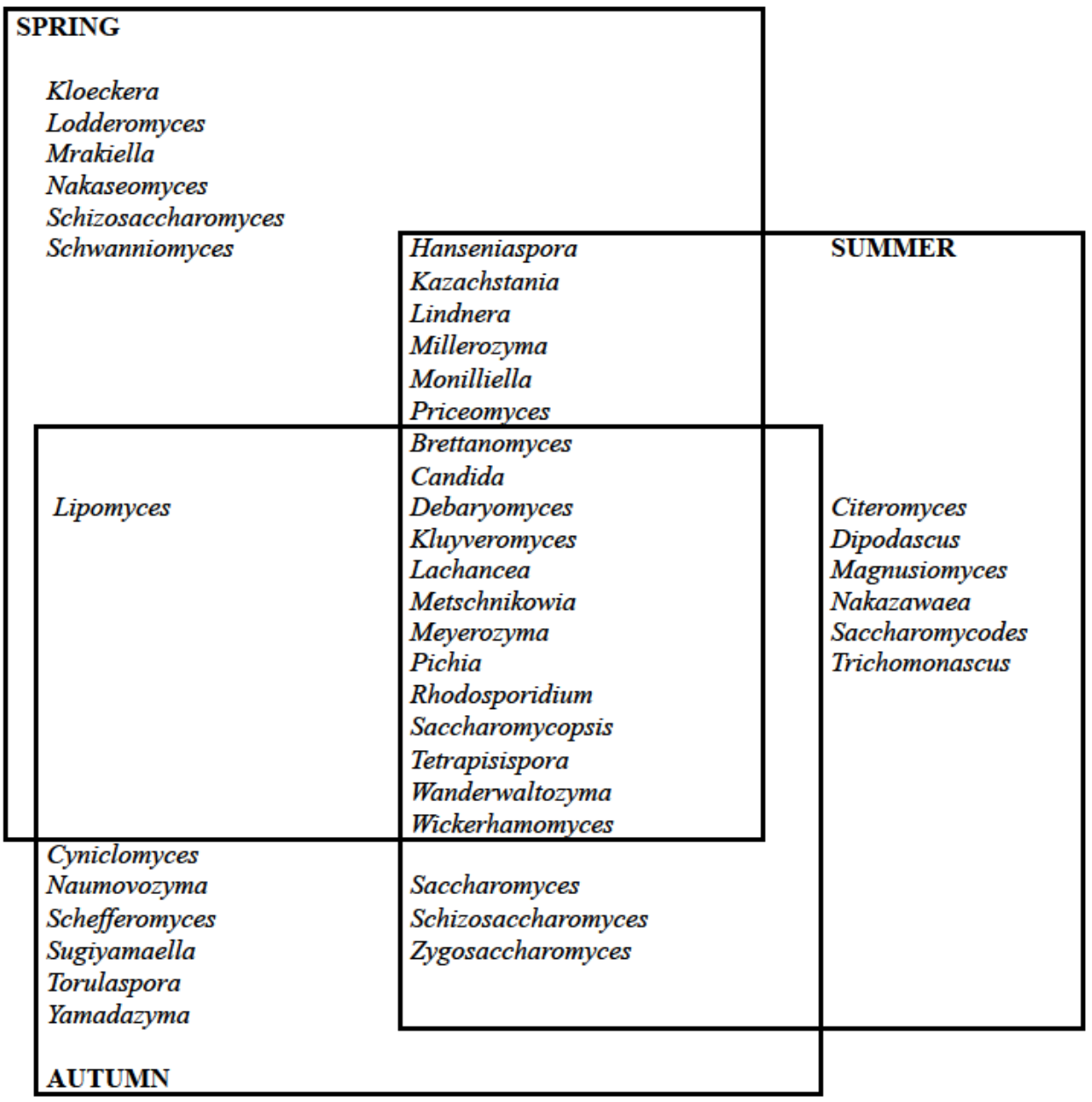

Fig. 2. Seasonal species diversity of yeast-like fungi.

A close correlation was identified between seasons of the year, a reservoir's trophic state and the nubmer of fungal cells in the surface water layer. The abundance of fungi increased from spring until autumn in moderately and highly eutrophized lakes. A reverse relationship was observed in the low trophic state lake (Tab. 3).

Table 3

Mean number of fungal cells per $1 \mathrm{dm}^{3}$ of water in individual seasons of the year in lakes with varying trophic states

\begin{tabular}{|c|c|c|c|c|}
\hline Lake & $\begin{array}{c}\text { Average number } \\
\text { of fungal cells } \\
\left(\mathrm{jtk}^{\prime} \mathrm{dm}^{3}\right)\end{array}$ & $\begin{array}{c}\text { Spring } \\
\left(\mathrm{jtk} / \mathrm{dm}^{3}\right)\end{array}$ & $\begin{array}{c}\text { Summer } \\
\left(\mathrm{jtk} / \mathrm{dm}^{3}\right)\end{array}$ & $\begin{array}{c}\text { Autumn } \\
\left(\mathrm{jtk} / \mathrm{dm}^{3}\right)\end{array}$ \\
\cline { 3 - 5 } & 3210 & 1600 & 1200 & 410 \\
\hline Tyrsko & 4000 & 800 & 1400 & 1800 \\
\hline Dhugie & 5480 & 880 & 2100 & 2500 \\
\hline Skanda & \multicolumn{3}{|c}{} \\
\hline
\end{tabular}




\section{DISCUSSION}

Our investigations confirm that yeast-like fungi tend to occur permanently in lakes. More species of fungi were identified in our study than in previous years (Dynowska 1995). This may be caused by different, more accurate isolation techniques, a greater number of samples and higher sampling frequency. Twenty two species of fungi were isolated from lake Skanda in a study conducted in the 1990s (Dynowska 1995) while 28 species were recorded in our study. Eleven species isolated by Dynowska (1995) in lake Skanda were also recorded in our study. These are potential pathogens (e.g., Candida albicans, Candida tropicalis, Meyerozyma guilliermondii, Saccharomycopsis capsularis or Magnusiomyces capitatus (formerly Trichosporon cutaneum)) whose occurrence in the water of a highly eutrophized lake is fully justified. Similar investigations were also conducted in lake Głębokie in Szczecin. It is a highly eutrophized lake and its parameters are similar to those of lake Skanda. Six of the eight species recorded in lake Głębokie (Biedunkiewicz et al. 2007) were recorded in the lakes in Olsztyn (Candida melibiosica, C. tropicalis, Pichia farinosa, P. polymorpha, Saccharomycodes ludwigii i Saccharomycopisis capsularis).

Candida albicans, Debaryomyces hanseni, Lindnera jadinii (formerly Pichia jadinii), Meyerozyma guilliermondii and Pichia membranifaciens were noted in the three lakes. These species are potential pathogens with broad-spectrum pathogenicity. Candida albicans has been treated as a pollution indicator in a variety of waters in the USA and Canada since the 1970s and it occurs when fresh municipal waste has been delivered (ASTM 2005). It is an opportunistic fungus which has a high pathogenic potential and quickly adapts to changing environmental conditions (Dynowska 1995).

It is disconcerting that potentially pathogenic species such as Candida albicans, Candida utilis (anamorphic stage of Lindnera jadinii) or Candida guilliermondii (anamorphic stage of Meyerozyma guilliermondii) occur in the lakes regardless of their trophic state. Water bodies containing these species can be a source of fungal infections, especially if bathing sites for use in summer are set up along the shores.

An inversely proportional correlation was observed between the eutrophication level and the number of fungal species. Only 28 species of fungi were recorded in a highly eutrophized lake, 40 in a weakly trophic lake and as many as 49 species in a low-trophic-state lake. This is consistent with the rule of increased species abundance and decreased species diversity dependent on the trophic state. While investigations were conducted over only one study season, a growth trend in the abundance of cells of microfungi corresponding with the eutrophication level, progressing from spring to autumn, was observed. The number of cells of microfungi isolated in our study was the highest in spring and the smallest in autumn only in the hydrologically closed lake Tyrsko, which is surrounded by a coniferous forest and has a very high maximum depth. A similar trend was observed in studies by Biedunkiewicz and Baranowska (2011) conducted five years ago.

Our investigations confirmed that both the species composition and the abundance of fungi in surface waters are correlated with a direct supply of biogens and resulting eutrophication. Similar observations were reported in a study conducted in a microphytic treatment plant in Nowa Słupia (Biedunkiewicz, Ozimek 2009) where 
the number of fungal cells recorded at the first stage of investigations (settlement tank, aeration chamber and reed beds) was similar to that noted in the highly eutrophic lake Skanda. A similar species composition of microfungi was also observed. Candida albicans, Candida guilliermondii, Candida glabrata, Candida tropicalis or Saccharomyces cerevisiae are some of the species noted in both the treatment plant and the lake.

Accelerated and progressing water eutorphication is mostly caused by an increased content of phosphorus derived from municipal and storm waste and of nitrogen derived chiefly from soil leaching. Phosphorus and, to a lesser extent, nitrogen contribute to the development of phytoplankton and littoral vascular vegetation. This leads to algal blooms and increased water transparency and, consequently, to oxygen deficits. As fungi are absolute anaerobes, oxygen deficiency does not limit their presence and may only partly limit multiplication of species already living in the water. If the diversity of organic matter increases, this may lead even to the occurrence of new species, very often expansive. Our results confirm this and show that the sanitary and epidemiological condition of water in selected lakes in Olsztyn is very bad.

\section{CONCLUSIONS}

As a permanent component of surface waters, yeast-like fungi can colonize waters of varying trophic states. They quickly adapt to dynamic conditions of the water environment which may be considered to be their natural reservoir.

Acknowledgements. The authors would like to thank an anonymous reviewer for valuable suggestions to improve this manuscript. Studies ${ }^{\star}$ were supported by the Ministry of Science and Higher Education (grant no. N N305 162239).

\section{REFERENCES}

ASTM D4249 - 83(2005) Standard Test Method for Enumeration of Candida albicans in Water.

Biedunkiewicz A. 2007. Grzyby w ocenie sanitarno-epidemiologicznej wybranego kapieliska. Diagnozowanie stanu środowiska, metody badawcze - prognozy. (In:) J. Garbacz (ed.) Prace Komisji Ekologii i Ochrony Środowiska Bydgoskiego Towarzystwa Naukowego 1: 107-121.

Biedunkiewicz A., Baranowska E. 2011. Yeast-like fungi as an element assessment of purity in lake Tyrsko In Olsztyn (Poland). Pol. J. Environ. Stud. 20 (2): 267-274.

Biedunkiewicz A., Ozimek T. 2009. Qualitative and quantitative changes of potentially pathogenic fungi in hydrophyte wastewater treatment plant (case study in Nowa Słupia, Poland). Pol. J. Environ. Stud. 18 (2): 161-166.

Biedunkiewicz A., Silicki A., Mazurkiewicz-Zapałowicz K. 2007. Yeast-like fungi in selected bath of Szczecin. Limnol. Rev. 3: 3-10

Biedunkiewicz-Ziomek A., Dynowska M. 2004. Candida dubliniensis Sullivan et al. - a new species in the human respiratory system. Acta Mycol. 39 (1): 7-11.

Dynowska M. 1995. Drożdże i grzyby drożdżopodobne, jako czynniki patogenne oraz bioidykatory ekosystemów wodnych. Studia I Materiały WSP w Olsztynie, 77.

Dynowska M., Biedunkiewicz A., Ejdys E. 2001. Pathogenic Yeast - Like Fungi with Bio - Indicator Properties. Pol. J. Environ. Stud. 10, Suppl. I - ECO - MED: 13-16. 
Dynowska M., Biedunkiewicz-Ziomek A., Kisicka I. 2005. Eco-physiological differentiation of yeast-like fungi from different aquatic ecosystems. Ecohydrology and Hydrobiology 5 (2): 147-153.

Kurtzman C.P., Fell J. W. 2000. The Yeast. A taxonomic study. 4th, Elsevier - Amsterdam.

Kurtzman C.P., Fell J.W., Boekhout T. 2011. The Yeast. A taksonomic study. $5^{\text {th }}$ ed. Elsevier - Tokyo.

Lodder J., Kreger-van Rij N.J.W. 1967. The Yeasts. A Taxonomic study. North Holland Publishing Company - Amsterdam

Lossow K., Gawrońska H., Mientki C., Łopata M., Wiśniewski G. 2005. Jeziora Olsztyna. Stan troficzny, zagrożenia. MAZUR PRINT - Olsztyn.

\section{Różnorodność gatunkowa grzybów drożdżoidalnych w wybranych jeziorach eutroficznych Olsztyna}

\section{Streszczenie}

Grzyby są stałym komponentem mikrobioty wód śródlądowych a każdy wzrost ich liczebności wiąże się najczęściej ze wzrastającą antropopresją i postępującym procesem eutrofizacji. Celem badań było określenie zróżnicowania taksonomicznego grzybów w trzech wybranych jeziorach Olsztyna, różniących się między sobą pod względem morfometrii, naturalnej odporności na degradację, stopniem zeutrofizowania i walorów przyrodniczych.

Badania prowadzono w 2011 roku, od wczesnej wiosny do późnej jesieni. Do szczegółowych analiz zastosowano metodę filtrów membranowych oraz metody hodowli i identyfikacji zalecane w diagnostycznych laboratoriach mykologicznych. Pobrano 120 prób wody z 20 stanowisk. Uzyskano 124 izolaty grzybów drożdżoidalnych, wśród których zidentyfikowano 84 gatunki z 39 rodzajów.

Stwierdzono ścisłą zależność między porami roku, trofią zbiornika a liczbą komórek grzybów w analizowanej warstwie wód powierzchniowych. Liczebność grzybów wzrastała od wiosny do jesieni w jeziorach umiarkowanie i silnie zeutrofizowanych. W jeziorze o niskiej trofii zaobserwowano odwrotną zależność. 